\title{
Time to end the political rhetoric on health tourism
}

The affiliation listed for the third author of this Letter, Gemma Bowsher, is incorrect (BMJ 2015;350:h2215, doi:10.1136/bmj. $\mathrm{h} 2215)$. She is a medical student at King's College London and the address is: King's College London, GKT School of Medical Education, Guy's Campus, London, SE1 1UL. 